\title{
Self-sufficient sensor for oxygen detection in packaging via radio-frequency identification
}

\author{
C. Weigel ${ }^{1}$, M. Schneider ${ }^{1, *}$, J. Schmitt ${ }^{1, * *}$, M. Hoffmann ${ }^{1}$, S. Kahl ${ }^{2}$, and R. Jurisch ${ }^{2}$ \\ ${ }^{1}$ Micromechanical Systems Group, IMN MacroNano ${ }^{\circledR}$, Technische Universität Ilmenau, Ilmenau, Germany \\ ${ }^{2}$ microsensys GmbH, Erfurt, Germany \\ * now at: Robert Bosch GmbH, Reutlingen, Germany \\ ** now at: Sonceboz Automotive SA, Sonceboz, Switzerland \\ Correspondence to: C. Weigel (christoph.weigel@tu-ilmenau.de)
}

Received: 3 November 2014 - Revised: 17 April 2015 - Accepted: 24 April 2015 - Published: 27 May 2015

\begin{abstract}
A new disposable radio-frequency identification (RFID) sensor for detecting oxygen in packages with a protective atmosphere is presented. For safety reasons and system costs in consumer packages, no battery or energy harvesting devices can be used. Each part of a package, especially in food packaging, must be completely safe even if it is swallowed. Several materials have been investigated that safely react with oxygen and thus change electrical parameters without the need of an additional energy supply. In particular linseed oil was tested, because it is known to react in oxygen-containing atmosphere from liquid to solid. Linseed oil is used not only as food but also as a key part in ecological paint coatings. A significant relative change of capacity was observed during linseed oil drying, which results in $-20 \%$ after $5 \mathrm{~h}$ and $-38 \%$ after $30 \mathrm{~h}$ at an oxygen concentration of 20.5 and $50 \%$ relative humidity, respectively. Pure unsaturated fatty acids were also tested in an oxygencontaining atmosphere and showed similar behaviour. The reaction speed is partially dependent on the level of unsaturation of fatty acids.

The oxygen sensor is coupled with an RFID front end with an internal charge time measurement unit for capacity determination. The combination of sensor element, sensitive material and RFID allows for biocompatible and save systems that indicate the presence of oxygen within a package.
\end{abstract}

\section{Introduction}

Metal oxide gas sensors are widely used for oxygen detection. Semiconducting metal oxide surfaces such as titanium oxide, zinc oxide and others show an oxygendependent conductivity (Kreisl, 2006). Electrochemical oxygen sensors for measuring liquids and gases are generally based on the amperometric principle, in which the current of a galvanic cell can be measured. One kind of amperometric oxygen sensor is the Clark electrode with liquid electrolyte, which is the most often used oxygen sensor at room temperature. In this case, the oxygen diffuses through a membrane into the liquid electrolyte and generates a current (Clark and Lyons, 1962; Otto, 2011). Furthermore, amperometric sensors with solid electrolyte, which are based on doped zirconia as an ion-conduction material between two electrodes, are employed as well (Tränkler and Obermeier, 1998; Kamp, 2003). Paramagnetic sensors require a heating wire and a permanent magnet. Inside the sensor, gas circulations occur through heating the gas molecules that cool down on the outer wall. As oxygen is paramagnetic it will be accelerated through magnetic repulsion to the wire and cools it at increased rate. The changing heater resistivity can be measured (Reichl, 1989). All these methods are suitable for continuous measurements of oxygen concentrations. Furthermore, those solutions usually require thermal activation energy (Xu et al., 2000) or an electrical potential difference that complicates the usage in self-sufficient systems. In paramagnetic sensors, temperatures of about $300^{\circ} \mathrm{C}$ are needed, and metal oxide sensors based on zinc oxide begin to absorb oxygen at temperatures above $120^{\circ} \mathrm{C}$ (Reichl, 1989). 
Additionally, those systems are complex, resulting in higher costs. These facts do not allow for the usage of sensing elements in packaging of mass products.

Due to cost and environmental constraints, sensing in end consumer packaging excludes energy sources such as batteries or energy harvesting systems. Additionally, hazardous substances could find a way into the packaged goods when those systems are damaged, e.g. during opening of the package.

Low-cost sensors for food application should be as simple as possible and preferentially set up from packaging or food ingredients. The usage of biocompatible substances is preferred. To read out the status, a wireless transmission via radio-frequency identification (RFID) is a suitable way for checking even larger quantities of packages at once. At least a simple identification of leaking packages is possible. Ideally the sensing should be irreversible at a defined oxygen concentration threshold.

\section{Concept of a self-sufficient oxygen sensor}

To realise self-sufficient, autonomous sensor elements, sensitive materials with low activation energy are required. Some organic materials show a change of conductivity at room temperature. Polyacetylene, polypropylene and polyphenylen sulfide are listed as important representatives. Most of them are assumed to be toxic or lead to irritation of respiratory tracts. The acceptability with regard to food packages is not without limitations (Sigma-Aldrich, 2013; de Moraes Porto, 2012).

But also unsaturated fatty acids are sensitive to reactions with oxygen. In nature many variations and grades of unsaturation exist, which influence the reaction speed at oxygen contact. Some of them are used not only in food but also for applications such as paints and lacquers, sometimes for centuries.

\subsection{Oxidation of fatty acids}

Fatty acids are aliphatic monocarboxylic acids that include one carboxyl group $(\mathrm{RC}(=\mathrm{O}) \mathrm{OH})$ and show a non-cyclic carbon skeleton. Natural acids appear in oils, fats and wax from animals and plants (McNaught, 1997). The classification depends on the binding of carbonic atoms and builds two groups: saturated and unsaturated fatty acids, in which double carbon bindings exists (Chow, 2008). The oxygen attacks preferentially these double bindings and causes internal chemical changes. Therefore, the number of unsaturated bindings is an indication of the reaction rate. Holman and Elmer (1947) showed that by measuring the absorbed amount of oxygen, the reaction kinetics depends on the degree of unsaturation. Acids with more than one unsaturated binding are, for example, linoleic acid (two double bindings), arachidonic acid (four double bindings) and docosahexaenoic acid (six double bindings).

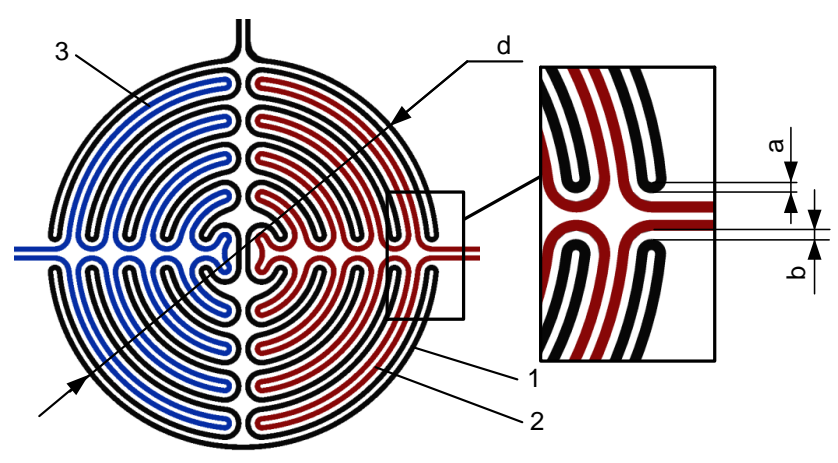

Figure 1. Electrode configuration for measuring capacity and resistivity including the ability to heat the active area (left: outer conductor (1) with a structure diameter $(d)$ to heat and two conductors $(2,3)$ for referencing and sensing; right: dimensions of circuit path width $(a)$ and distance $(b))$.

The reaction with oxygen was analysed in a variety of publications. The following description is, for example, given in Kuang Chow (2007):

start reaction: $\quad \mathrm{RH} \rightarrow \mathrm{R}$,

propagation: $\mathrm{R} \cdot+\mathrm{O}_{2} \rightarrow \mathrm{ROO}$,

$$
\mathrm{ROO}+\mathrm{RH} \rightarrow \mathrm{ROOH}+\mathrm{R},
$$

termination: $\quad 2 \mathrm{ROO} \rightarrow$ non radical products $+\mathrm{O}_{2}$.

RH represents multiple unsaturated fatty acid molecules, which lead to a creation of radicals $\mathrm{R}$. By the availability of oxygen, $\mathrm{R}$ reacts quickly and forms the hyperperoxyl radical $\mathrm{ROO}$, which builds the stable hyperperoxide $\mathrm{ROOH}$.

For fast reactions, the percentage of multiple unsaturated radical molecules is essential. Dubois et al. (2008) shows some natural oils divided in three groups of fatty acids. Linseed oil shows a high percentage of multiple unsaturated bindings, which is an indication of short reaction times. Furthermore, after the oxidative polymerisation process, linseed oil approaches a stable condition by forming longchain molecules. This is contrary to technical oils, which decompose in a reverse direction. During the oxidation, first the volume of linseed oil increases and finally decreases when the polymerisation starts. The reaction time depends on a multitude of ambient conditions such as humidity, temperature and oxygen concentration.

\subsection{Sensor element}

\subsubsection{Design and fabrication of a combined measuring and heating system}

The sensor electrodes should be designed for high sensitivity. For this reason, an optimised ratio of large electrode area and small gap size should be obtained for an almost simple 


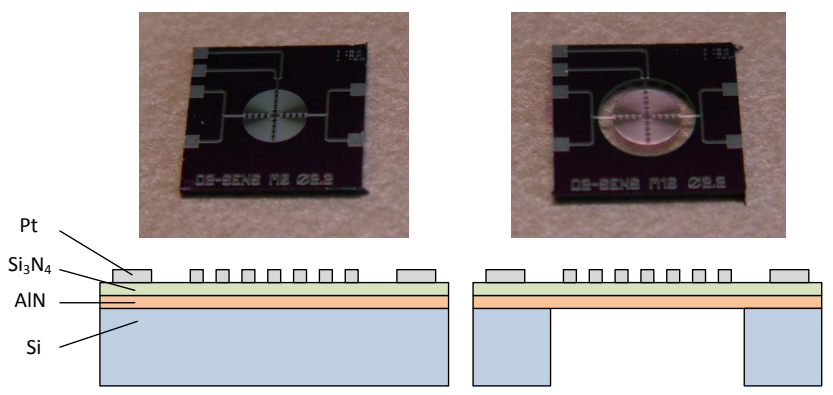

Figure 2. Cross sectional view and fabricated sensors with a diameter of $2.2 \mathrm{~mm}$ on bulk silicon (left) and on an AlN membrane (right).

capacity measurement. In order to achieve reliable results, structures for sensing and referencing and the possibility for heating the structures to a reference temperature are included.

The designed interdigital structure is shown in Fig. 1, which has an aspect ratio from circuit path width $(a)$ to distance $(b)$ of $4: 3$, and two separate structures for measuring and referencing including the option to heat the sensor.

This design is able to measure capacity as well as resistivity values in a three-wire configuration. The outer conductor (1) can be used as a heating element, too. This allows for reaching stable and homogenous temperature distribution over the whole structure, if required. Both symmetrical inner conductors can be used for measuring (2) and referencing (3) for the electrical characterisation. The heating and measuring elements are manufactured from a single metallisation layer and allow, subsequently, for a simple fabrication process.

In all, $<100>$ silicon wafers $(1-20 \Omega \mathrm{cm})$ are used for the demonstrator. The isolation is performed by using a $500 \mathrm{~nm}$ thick aluminium nitride (AlN) and an additional $250 \mathrm{~nm}$ silicon nitride $\left(\mathrm{Si}_{3} \mathrm{~N}_{4}\right)$ film. The platinum conductors are fabricated in a lift-off process. Aluminium (Al) conductors are suitable, if heating is not required. A second aluminium layer is applied at the pad areas for improved wire bonding. In principle, the same structure can easily be reproduced on flexible foils with any kind of metallisation process.

Furthermore, sensing devices can be fabricated on membranes by backside deep reactive ion etching (DRIE). This allows for low energy consumption if sensitive materials with higher activation energy require heating. Figure 2 shows devices with and without a membrane.

\subsubsection{Characterisation and experiments with sensitive materials for oxygen detection}

The initial resistivity and capacity of the conductors were characterized. In Fig. 3, the capacity for different layouts listed in Table 1 at different frequencies is shown. The

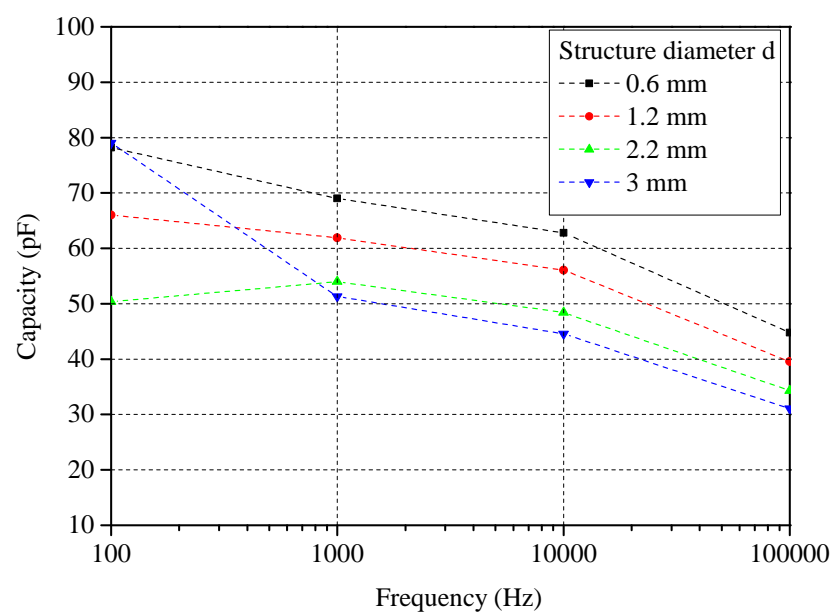

Figure 3. Characterisation of capacity at selected structure diameters $(d)$ with different path widths $(a)$ and distances $(b)$ listed in Table 1 in the frequency range of $100-100 \mathrm{kHz}$ (without sensitive material).

Electrical Potential $(V)$, Height $(\mu \mathrm{m})$

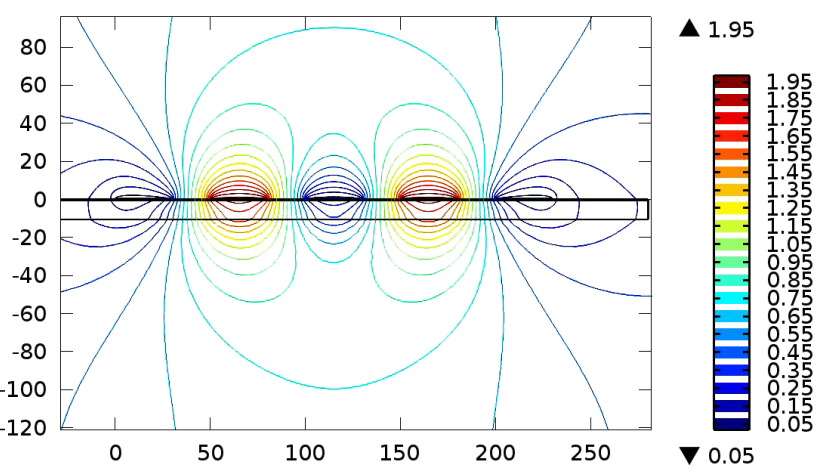

Figure 4. Simplified stray field model of structure with $2.2 \mathrm{~mm}$ diameter for simulating the area of high electrical fields (in this case about $50 \mu \mathrm{m}$ above the electrodes).

ambient gas during this process was nitrogen with a relative dielectric constant $\varepsilon_{\mathrm{R}}=1$.

The sensitive material is applied onto the sensing structure. The permittivity increases when the fatty acids/oils are introduced. In literature, values between 3.2 and 3.5 are reported for linseed oil. The resulting capacity splits up into the substrate capacity and effect due to the sensitive material. The sensitive material should preferentially cover the volume of the capacitive stray field to achieve reliable changes due to the oxidation. Therefore, COMSOL Multiphysics simulations were performed to determinate the required volume and thickness of the sensitive layer (Fig. 4).

Table 1 shows simulated stray field heights for different layouts that have to be coated with sensitive material. The dispensing of the oils can be done in ambient air and the achieved capacity is measured. The delay before electrical 
Table 1. Structure sizes and simulated stray field height of the capacitor.

\begin{tabular}{rrrr}
\hline Structure diameter $(d)$ & Path width $(a)$ & Path distance $(b)$ & Stray field height \\
\hline $0.6 \mathrm{~mm}$ & $8 \mu \mathrm{m}$ & $5 \mu \mathrm{m}$ & $10 \mu \mathrm{m}$ \\
$1.2 \mathrm{~mm}$ & $16 \mu \mathrm{m}$ & $10 \mu \mathrm{m}$ & $20 \mu \mathrm{m}$ \\
$2.2 \mathrm{~mm}$ & $30 \mu \mathrm{m}$ & $20 \mu \mathrm{m}$ & $40 \mu \mathrm{m}$ \\
$3 \mathrm{~mm}$ & $40 \mu \mathrm{m}$ & $30 \mu \mathrm{m}$ & $60 \mu \mathrm{m}$ \\
\hline
\end{tabular}

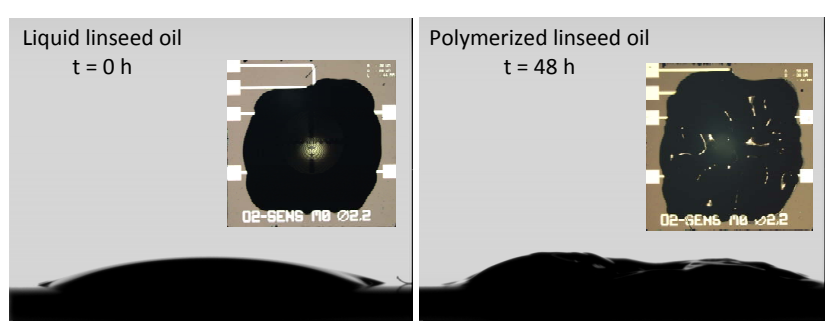

Figure 5. Sensor (structure diameter $d=2.2 \mathrm{~mm}$ ) with liquid linseed oil (left) at the beginning and after polymerisation to linoxyn (right).

parameters start to drift depending on the fatty acids and it is usually long enough (more than $10 \mathrm{~min}$ ) to place the sensor into the measuring set-up. Linseed oil is a liquid. In an inert atmosphere, the capacity does not change and it stays liquid. If oxygen is present the unsaturated fatty acids start a reaction and consume oxygen molecules. The permittivity and thus the measured capacity changes during the reaction and equals a stable state after the polymerisation is finished. Figure 5 shows linseed oil before and after the oxidative polymerisation process on a sensor device.

Figure 6 shows the changes of capacity of a structure with $3 \mathrm{~mm}$ diameter at ambient conditions ( $21 \%$ oxygen concentration), $293 \mathrm{~K}$ and a relative humidity of $50 \%$ in a frequency range between $100 \mathrm{~Hz}$ and $100 \mathrm{kHz}$. The capacity was determined without, with liquid and with solidified linseed oil in steady state. It can clearly be seen, that the solidified oil has a significantly lower dielectric constant as compared to the liquid state. Due to cross-linking of the fatty acids the reduction of the amount of polar groups causes a reduced dielectric constant.

Further experiments have been performed at a quasi-static state as the measurement principle of the RFID interface uses a similar method. Thus, a voltage ramp is used and the charging current is measured once an hour.

It was confirmed that the reaction speed depends on the humidity and concentration. Therefore, a set-up that allows for varying the concentration of oxygen, humidity and temperature in a measuring chamber is needed to achieve defined conditions. The concentration of oxygen is adjusted by using mass flow controllers for pure nitrogen and synthetic air. Humidity is adjusted by using a bubbler and a humidity sensor for control. Additionally, the chamber

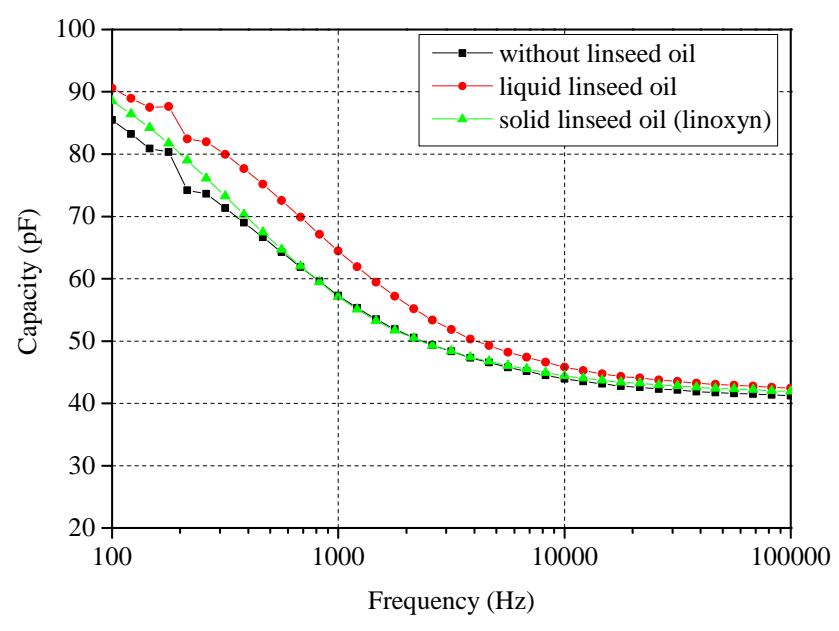

Figure 6. Capacity of a structure with a diameter $(d)$ of $3 \mathrm{~mm}$ ( $a=40 \mu \mathrm{m}, b=30 \mu \mathrm{m}$ ) in a frequency range of $100 \mathrm{~Hz}$ to $100 \mathrm{kHz}$, at conditions with and without solid and liquid linseed oil.

can be heated. The oxygen concentration is measured by using the oxygen sensor Mettler Toledo InPro 6800 G. Furthermore, sensors for pressure and temperature are included and allow for controlling the conditions inside the measurement chamber. Figure 7 shows a simplified scheme of the measurement set-up. Experiments are driven by a LabVIEW program, which allows for the setting and saving of the data. Sensors under test are assembled on a carrier module that connects sensor devices with a reusable RFID system.

In Fig. 8, measured capacity vs. time at oxygen concentrations of $1,10.25$ and $20.5 \%$ are shown at $293 \mathrm{~K}$ and $0 \%$ relative humidity. In a second study, the influence of the moisture at constant oxygen concentration is presented $(20.5 \%$ oxygen concentration, $293 \mathrm{~K})$. High humidity causes, as expected, an increase of capacity due to the high dielectric constant of water molecules. Due to polymerisation the influence of water decreases and results in a significant decrease of capacity.

Depending on the concentration of oxygen and moisture, the oxidative polymerisation requires a distinct time. Low oxygen concentrations as well as high humidity slow down the reaction.

The reaction can be forced by a higher surface-to-volume ratio as achieved by spin coating or by using materials 


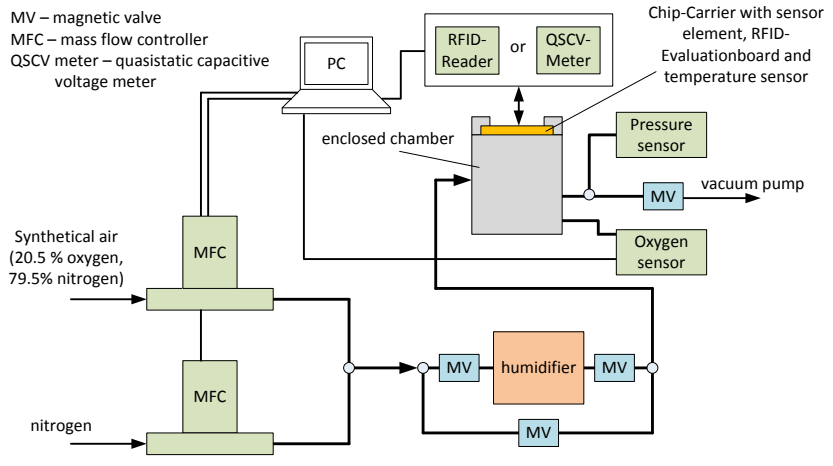

Figure 7. Simplified diagram of the test station with magnetic valves (MV) for flow path selection, mass flow controller (MFC) and sensor element with connected read-out devices via RFID and quasi-static capacitive voltage metre (QSCV).

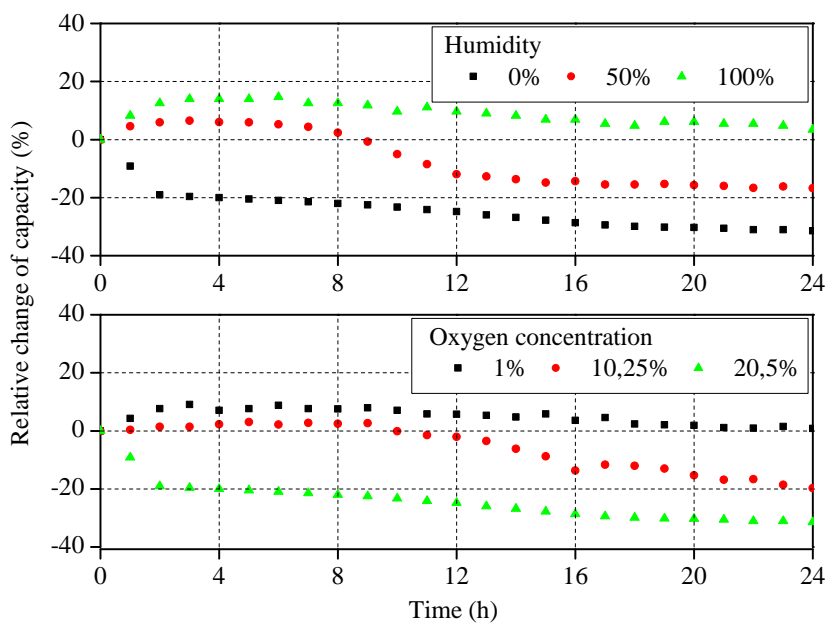

Figure 8. Relative change of capacity by varying the humidity (* high humidity, non-condensing) under constant oxygen concentration at $20.5 \%$ (top) and constant humidity at $0 \%$ at various oxygen concentrations (bottom) for a structure diameter $(d)$ of $3 \mathrm{~mm}$ with a path width $(a)$ and a distance $(b)$ of 40 and $30 \mu \mathrm{m}$.

with polyunsaturated fatty acids. Also docosahexaenoic acid ethyl ester (DHA-EE), arachidonic acid and linoleic acid that have 6,4 and 2 double bindings, respectively, have been tested. These are purified ingredients of linseed and tuna oil. Their reaction rate is more reproducible than that of the natural linseed oil with naturally varying contents of fatty acids. Figure 9 illustrates the relative change of capacity in ambient air ( $21 \%$ oxygen, $293 \mathrm{~K}, 50 \%$ relative humidity). Polyunsaturated fatty acids such as DHA-EE show a faster reaction as compared to linoleic acid. A volume of about $0.5 \mu \mathrm{L}$ DHA-EE applied to a $3 \mathrm{~mm}$ structure shows a steadystate condition after $25 \mathrm{~h}$. Therefore, a variable sensitivity against oxygen in packages can be achieved.

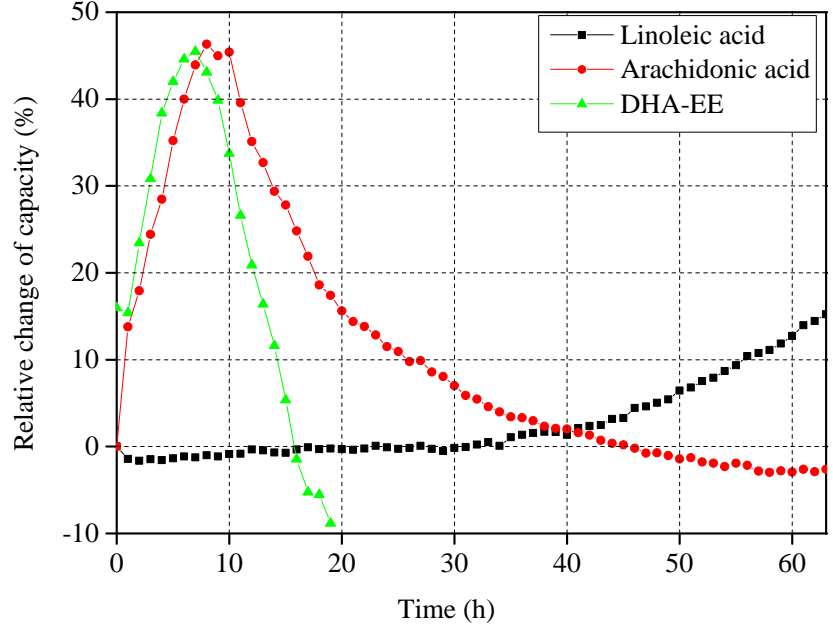

Figure 9. Time-dependent changes of capacity through the oxidation of fatty acids with various unsaturation ions (linoleic acid with 2, arachidonic acid with four and DHA-EE with six unsaturated bindings).

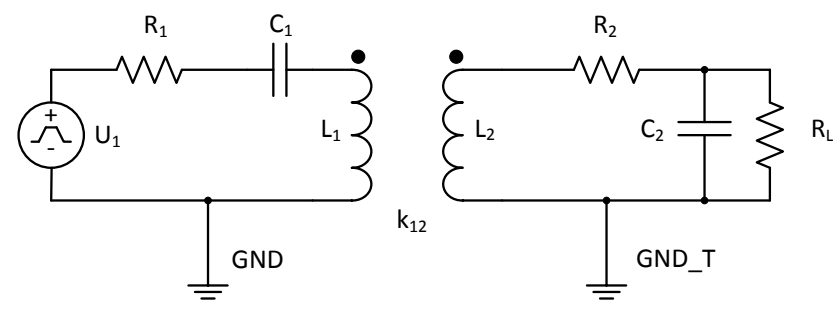

Figure 10. The oscillator circuits of the RFID reader and the transponder with connected sensor element described with the load resistance $R_{\mathrm{L}}$ as well as $L_{1}$ and $L_{2}$, which represent the transmitter and the transponder antenna.

\section{Connection between sensor element and RFID evaluation unit}

A suitable interface between the sensor element and RFID is required for a stable energy supply and data communication. Theoretical investigations for the power transmission have been performed. Starting point was the equivalent circuit and the complex equation approach in Finkenzeller (2006). The transmitter is based on a resonant antenna circuit and a resistive load. The sensor element consists of a resonator connected to the sensor, which represents the load. The equivalent circuit is shown in Fig. 10.

The transfer function between the output voltage $U_{2}$ and the input $U_{1}$ depends on the coupling factor between both oscillator circuits as well as their figure of merit and the load resistance. The load resistance also determines the available current for the sensing element. A load of $1 \mathrm{k} \Omega$ corresponds to a current of about $2 \mathrm{~mA}$. 


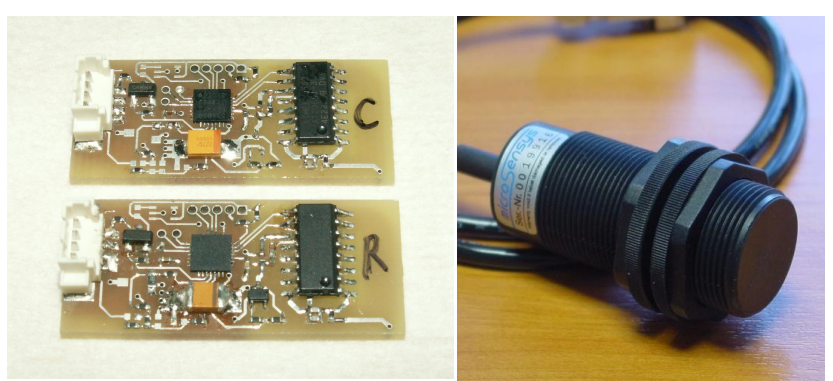

Figure 11. RFID-evaluation boards with front end controller for resistive and capacitive readout (left) and the used M30-Head for connection via USB to a processing unit (right).

\subsection{Used RFID hardware}

The oxygen sensor is connected to a commercial RFID front end from microsensys $\mathrm{GmbH}$. For the sensor transponder, an interface working at $13.56 \mathrm{MHz}$ was developed. Because of the required power for the transducer and the controller, a high-frequency RFID interface is preferred, which was built according to the ISO14443 type B standard. Therefore, the figure of merit of reader circuit and transponder was adjusted for an optimal bandwidth by decreasing the figure of merit and maximum field strength. The resonance frequency of both oscillating circuits is optimized for the ability to connect other reading systems from microsensys $\mathrm{GmbH}$ and for a wide functional range of the system.

Here, the so-called "M30-Head" was chosen and special commands for the transponder were integrated. The protocol is based on the $\mathrm{iID}^{\circledR}$ 3000Pro protocol and complements this in order to achieve more flexibility. The sensor adaption is done by the transponder controller. Figure 11 shows the developed RFID transponder for resistive and capacitive read-out and the used M30-Head.

The transponder consists of a commercial RFID front end iID-L ${ }^{\circledR}$ with voltage output, a microcontroller with integrated analogue-digital converter, and a unit to measure capacities. As the interface between front end and controller, a serial bus interface for data exchange is used. The output of the front end supplies a current of $2 \mathrm{~mA}$ for the sensor, e.g. for heating. For measuring the signal of the sensor, a high resistance input is used to analyse an analogue voltage as for measuring capacities. The design of the electronic compounds is optimised for a capacitance range between 50 and $90 \mathrm{pF}$, based on the measurements shown before. Figure 12 shows the block diagram of the used transponder.

\subsection{Measuring principle of the interface}

For measuring capacitive changes, two approaches can be used:

- A capacity-to-digital converter. Actually, this type is not available for this low voltage and low power application.

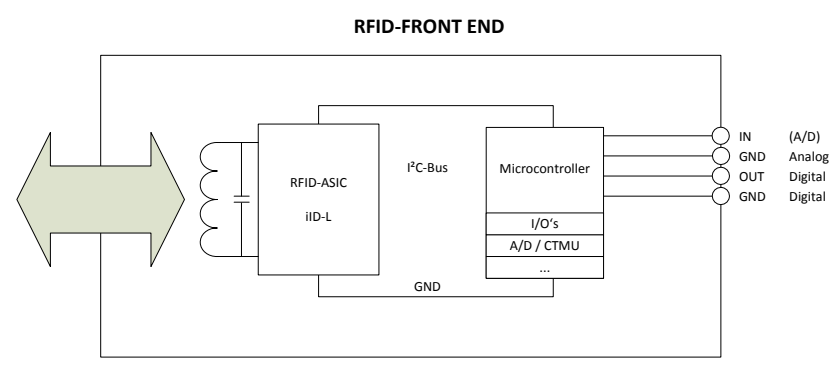

Figure 12. Block diagram of the transponder with integrated units for signal analysis and connections for the sensor element.

- Charge time measurement unit (CTMU), which is already available in the microcontroller. Thereby, a constant, adjustable current is injected for a defined time and the potential is read out as a measure of the capacitance.

The measurement cycle is as follows: the capacitor is discharged before each measurement. The connected capacity $C$ can be calculated from the current $I$ following Eq. (5)

$C=I \cdot \frac{\mathrm{d} U}{\mathrm{~d} t}$.

Assuming a starting voltage $U$ of $0 \mathrm{~V}$ at a discharged capacitor, correlation Eq. (6) can be applied if the time period $t$, the current $I$ and the measured voltage $U$ are known.

$C=\frac{I \cdot t}{U}$

For final calculation of the capacitance $C$ by using the output signal of the analogue-to-digital converter including internal and parasitical capacitance, the formula Eq. (7) can be assumed:

$C[\mathrm{pF}]=\frac{I_{\mathrm{c}} \cdot t_{\mathrm{c}}}{\mathrm{ADC} \cdot \frac{U_{\mathrm{cc}}}{1024}}-C_{\mathrm{int}}$.

The capacitance $C_{\text {int }}$ is constant, which is defined by parasitic capacitance of the electronic circuit. It is empirically determined. $I_{\mathrm{c}}$ is the charging current and $t_{\mathrm{c}}$ the charging time, which are set to $0.55 \mu \mathrm{A}$ and $200 \mu \mathrm{s}$, respectively. "ADC" is the measured digital value. The transponder measures relative to a reference value, which is recorded before starting the measurement. A calibration of the current source and the internal series capacity is not necessary. For the sensor adaption, a test firmware was programmed, which controls the operating voltage. Furthermore, the transponder checks the collected data via RFID-ASIC interface for consistency. After that, the potential and the capacitive value are memorised, handled and the timing is set according to the measured values. 

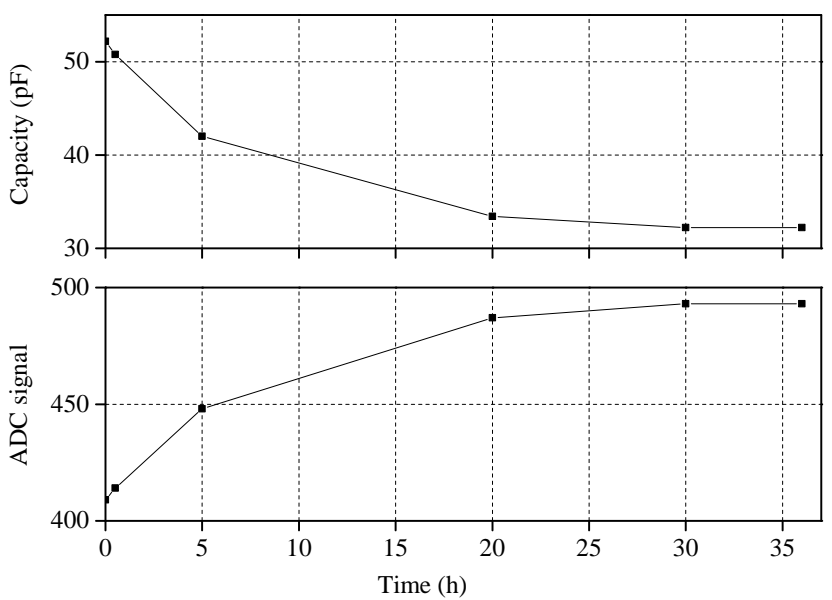

Figure 13. Measured capacity and corresponding ADC values from RFID transponder for a sensor with linseed oil (293 K, $0 \%$ rel. humidity) for a structure diameter of $3 \mathrm{~mm}$.

\subsection{Connection between sensor chip and transponder}

The sensor was connected to the RFID transponder. First, the value without sensitive layer was read out. The application of the material causes changes in the capacity. Thus, the value of the $\mathrm{AD}$ converter decreases. During the reaction time, the ADC value increases. After polymerisation it nearly reaches the value without sensitive material. The tests were performed at different sensor sizes. Larger sensors show higher changes and the evaluation is more accurate. By using Eq. (7), the ADC values can be converted into capacitive values with the typical voltage $U_{\mathrm{CC}}$ of $2.35 \mathrm{~V}$. In the following Table 2, data for the investigated test structures are given in ADC and real capacitive values.

Furthermore, the behaviour of linseed oil over time was recorded. After $5 \mathrm{~h}$, the RFID interface shows significant changes. After this time, damages of the package can be detected. By using other fatty acids, a much faster response is possible between the incidence of oxygen and measurable changes. The measured results of linseed oil are shown in Fig. 13.

Hence this demonstrates the applicability of these kind of sensors in combination with an RFID evaluation unit to observe the increase of oxygen in a protective gas atmosphere.

\section{Conclusions}

A new system concept of self-sufficient oxygen sensors is demonstrated, which records oxygen contamination without external energy. The intended application of the sensor is a threshold statement if unwanted oxygen access has occurred to the atmosphere under test. This is of interest for pharmaceuticals, for food under protective atmosphere and for industrial products such as oxygen-sensitive chemicals.
Table 2. Observed ADC and real capacity values of different chip sizes with liquid and solid linseed oil $\left(U_{\mathrm{cc}}=2.35 \mathrm{~V}\right)$.

\begin{tabular}{lrr|rr|rr}
\hline \multirow{2}{*}{$\begin{array}{l}\text { Membrane } \\
\text { size }\end{array}$} & \multicolumn{2}{c|}{ without } & \multicolumn{2}{c|}{ liquid } & \multicolumn{2}{c}{ solid } \\
\cline { 2 - 7 } & ADC & real & ADC & real & ADC & real \\
\hline $0.6 \mathrm{~mm}$ & 715 & $2 \mathrm{pF}$ & 700 & $4 \mathrm{pF}$ & 708 & $2.7 \mathrm{pF}$ \\
$1.2 \mathrm{~mm}$ & 639 & $10 \mathrm{pF}$ & 616 & $13 \mathrm{pF}$ & 639 & $10 \mathrm{pF}$ \\
$2.2 \mathrm{~mm}$ & 469 & $37 \mathrm{pF}$ & 456 & $40 \mathrm{pF}$ & 468 & $37 \mathrm{pF}$ \\
$3 \mathrm{~mm}$ & 493 & $32 \mathrm{pF}$ & 409 & $52 \mathrm{pF}$ & 493 & $32 \mathrm{pF}$ \\
\hline
\end{tabular}

Therefore, materials which are safely reacting with oxygen were analysed. Unsaturated fatty acids, for example in linseed oil, polymerize in oxygen-containing atmospheres and have been proven to be suitable candidates. The sensitive material was applied to a capacitor and changes its electrical parameter during the polymerisation. Linseed oil shows significant changes of its dielectric constant of about nearly $40 \%$ depending on the ambient conditions. To reach lower response times, fatty acids with more double bonds are suitable at a comparable behaviour than linseed oil. Ambient conditions such as humidity and temperature influence the reaction speed, but do not significantly influence the threshold statement.

External energy is only needed during the readout process of the sensor element. This allows for the integration in packages without batteries or energy harvesting. A wireless readout is usually required as the sensor has to be inside the supervised package. Therefore, an RFID interface was developed that allows for the wireless read-out of the capacitance.

The irreversible chemical reaction guarantees high protection against manipulation. Furthermore, the design is simple and thus it allows for low production costs for mass market applications. A miniaturisation and integration are aspired to allow for the usage in smart transponder housings comparable to D14 from microsensys $\mathrm{GmbH}$. The dimension of such a system are only $14 \mathrm{~mm}$ in diameter and $2 \mathrm{~mm}$ in height. Also a fabrication on foils can be realised, which would allow for the direct integration in packages.

Acknowledgements. This work was supported by the Federal Ministry of Education and Research (BMBF) by the project "O2Sens - Niedrigenergie-Sensor zum Nachweis von Sauerstoff in Verpackungen mittels RFID” (contact no. 16SV5277).

The authors thank Jutta Uziel, Birgitt Hartmann, Joachim Döll and Ilona Marquardt from the Technische Universität Ilmenau for supporting the fabrication process and Yahia Cheriguen for his support by creating the stray field simulation models. Furthermore, we thank Ingo Hörselmann from the Technische Universität Ilmenau for his support in electrical measurements.

Edited by: J. Zosel

Reviewed by: two anonymous referees 


\section{References}

Chow, C. K. (Ed.): Fatty acids in foods and their health implications, 3rd Edn., Food Sci. Technol., CRC Press, Boca Raton, USA, 1281 pp., 2008.

Clark, L. C. and Lyons, C.: Electrode Systems For Continuous Monitoring In Cardiovascular Surgery, Ann. Ny. Acad. Sci., 102, 29-45, doi:10.1111/j.1749-6632.1962.tb13623.x, 1962.

de Moraes Porto, I. C. C.: Polymer Biocompatibility, Polymerization, edited by: de Souza Gomes, A., InTech, doi:10.5772/47786, 2012.

Dubois, V., Breton, S., Linder, M., Fanni, J., and Parmentier, M.: Proposition de classement des sources végétales d'acides gras en fonction de leur profil nutritionnel, OCL, 15, 56-75, doi:10.1051/ocl.2008.0163, 2008.

Finkenzeller, K.: RFID-Handbuch: Grundlagen und praktische Anwendungen induktiver Funkanlagen, Transponder und kontaktloser Chipkarten, 4th Edn., Hanser, München, Wien, 2006.

Holman, R. T. and Elmer, O. C.: The rates of oxidation of unsaturated fatty acids and esters, J. Am. Oil Chem. Soc., 24, 127-129, doi:10.1007/BF02643258, 1947.

Kamp, B.: Beiträge zur Sensorik redox-aktiver Gase, $\mathrm{PhD}$ Thesis, University of Stuttgart, Stuttgart, Germany, available at: http://elib.uni-stuttgart.de/opus/volltexte/2003/1241/ (23 October 2014), 2003.
Kreisl, P.: Mikromechanische Plattform für Metalloxidund MOS-Gassensoren auf SOI-Basis, Berichte aus der Mikrosystemtechnik, Shaker, Aachen, Germany, 168 pp., 2006.

Kuang Chow, C.: Biological Effects of Oxidized Fatty Acids, 3rd. Edn., CRC Press, 855-878, doi:10.1201/9781420006902.ch36, 2007.

McNaught, A. D. and Wilkinson, A.: Compendium of chemical terminology: IUPAC recommendations, 2nd Edn., Blackwell Science, Oxford, UK, Malden, MA, USA, VII, 450 pp., 1997.

Otto, M.: Analytische Chemie, 4th Edn., Bachelor, Wiley-VCH, 2011.

Reichl, H.: Halbleitersensoren: Prinzipien, Entwicklungsstand, Technologien, Anwendungsmöglichkeiten, Kontakt \& Studium, 251, Expert Verlag, Renningen, Germany, 1989.

Sigma-Aldrich: Safety Data Sheet Polypyrrole, Version 5.1, available at: http://www.sigmaaldrich.com/MSDS/ MSDS/DisplayMSDSPage.do?country=DE\&language $=$ EN-generic\&productNumber $=577030 \&$ brand= ALDRICH\&PageToGoToURL=\%2Fsafety-center.html, 2014.

Tränkler, H.-R. and Obermeier, E.: Sensortechnik, Springer Berlin Heidelberg, Berlin, Heidelberg, Germany, 1116-1119, 1998.

$\mathrm{Xu}$, Y., Zhou, X., and Sorensen, O.: Oxygen sensors based on semiconducting metal oxides: an overview, Sensor. Actuat. BChem., 65, 2-4, doi:10.1016/S0925-4005(99)00421-9, 2000. 